ARTICLE

Received 21 Sep 2015 | Accepted 31 Aug 2016 | Published 13 Oct 2016

DOl: $10.1038 /$ ncomms13117

OPEN

\title{
Chiral optical response of planar and symmetric nanotrimers enabled by heteromaterial selection
}

Peter Banzer 1,2,3,4, Paweł Woźniak ${ }^{1,2,4}$, Uwe Mick ${ }^{1,2,4}$, Israel De Leon ${ }^{3,4,5}$ \& Robert W. Boyd $3,4,6$

Chirality is an intriguing property of certain molecules, materials or artificial nanostructures, which allows them to interact with the spin angular momentum of the impinging light field. Due to their chiral geometry, they can distinguish between left- and right-hand circular polarization states or convert them into each other. Here we introduce an approach towards optical chirality, which is observed in individual two-dimensional and geometrically mirror-symmetric nanostructures. In this scheme, the chiral optical response is induced by the chosen heterogeneous material composition of a particle assembly and the corresponding resonance behaviour of the constituents it is built from, which breaks the symmetry of the system. As a proof of principle, we investigate such a structure composed of individual silicon and gold nanoparticles both experimentally, as well as numerically. Our proposed concept constitutes an approach for designing two-dimensional chiral media tailored at the nanoscale, allowing for high tunability of their optical response.

\footnotetext{
${ }^{1}$ Max Planck Institute for the Science of Light, Guenther-Scharowsky-Straße 1, D-91058 Erlangen, Germany. ${ }^{2}$ Institute of Optics, Information and Photonics, University Erlangen-Nuremberg, Staudtstrasse 7/B2, D-91058 Erlangen, Germany. ${ }^{3}$ Department of Physics, University of Ottawa, 25 Templeton, Ottawa, Ontario, Canada K1N 6N5. ${ }^{4}$ Max Planck University of Ottawa Centre for Extreme and Quantum Photonics, University of Ottawa, 25 Templeton Street, Ottawa, Ontario, Canada K1N 6N5. ${ }^{5}$ School of Engineering and Sciences, Tecnológico de Monterrey, Monterrey, Nuevo León 64849 , Mexico. ${ }^{6}$ Institute of Optics, University of Rochester, Rochester, New York 14627, USA. Correspondence and requests for materials should be addressed to P.B.

(email: peter.banzer@mpl.mpg.de).
} 
O ptical chirality is a well-known phenomenon that has been intensely studied in the past and still attracts tremendous attention, especially in the field of modern optics. Optically chiral media possess a sense of handedness that allows them to interact differently with left- and right-handed circularly polarized light, and gives rise to important optical phenomena such as polarization rotation and circular dichroism (CD). Natural chiral media are composed of chiral molecules. The chiral optical response of many chiral molecules is understood as a direct consequence of their three-dimensional (3D) chiral geometry, which does not allow for a superposition of the molecule's chiral structure with its mirror-image. Nowadays, modern micro- and nano-fabrication techniques allow for the creation of elaborately and artistically shaped structures, such as $3 \mathrm{D}$ helical antennas ${ }^{1-7}$. Such structures exhibit a strong chiral response, especially when arranged into dense lattices. In particular, microscopic or nanoscopic helices are a prototypical example of artificial chiral structures, following one of mother nature's design recipes for chiral molecules. Their chiral optical response can be tuned or artificially created by controlling their size, the number of pitches or twists around the central axis and other geometric parameters. In contrast to their molecular archetypes, which are orders of magnitudes smaller than the wavelength of light, artificial chiral structures are comparable in size with the wavelength. As a consequence, the instantaneous electric field points into different transverse directions at different cross-sections along the helical object. Hence, such artificial structures may exhibit a richer resonance behaviour, which manifests itself in a large number of supported resonant (higher-order) modes and a stronger chiral response. Reviews on optical chirality and optically chiral nanostructures have been presented recently ${ }^{8,9}$.

It also has been shown that a similar chiral optical behaviour can result from a $3 \mathrm{D}$ chiral or layered arrangement of achiral nanoparticles, such as nanospheres, split-ring resonators or similar ${ }^{3,8,10-17}$. To showcase a comprehensible example here, one might think about an ensemble of nanospheres following a helical trace in $3 \mathrm{D}$-space $\mathrm{s}^{3,8,13-16}$. The resulting particle ensembles exhibit an intrinsically chiral geometry when effectively considered as a single object.

Truly planar or two-dimensional structures can also show a chiral optical response. Similar to the aforementioned 3D-chiral approaches, planar structures obtain their chiral optical properties by their lack of in-plane mirror-symmetry. Prominent examples are S- and L-shaped antennas or gammadion-type nanostructures ${ }^{18-27}$. Many such examples have been discussed in literature. Nonetheless, the full capabilities and underlying processes of chiral light-matter interaction, especially for planar chiral nanostructures, haven't yet been fully explored.

An alternative route has been proposed to obtain a chiral optical response from a two-dimensional nanostructure, which is based on so-called extrinsic or pseudo-chirality $22,28-31$. In contrast to the intrinsically geometrically chiral structures discussed so far, the concept of extrinsic chirality allows for a chiral optical response of symmetric and achiral nanostructures. In this scheme, an array of achiral nanostructures, such as split-ring resonators, is illuminated under oblique incidence. In this situation, the structures are not super-imposable with their mirror-images anymore with respect to a rotation restricted to a plane perpendicular to the propagation direction of the impinging light, rendering them chiral.

It is worth noting here that the majority of the abovementioned systems were comprises lattices (metasurfaces) of individual nanostructures or were investigated in an inhomogeneous environment (on substrates). Both settings are known for being capable of enhancing the chiral optical effect.
For instance, the presence of a substrate breaks the symmetry of the system along the surface normal, hence enhancing the chiral effect by making the lower and upper surface of a two-dimensional structure distinguishable.

Yet another approach towards optical chirality, which is of particular importance for this Letter, is the use of heterogeneous material compositions, similar to chiral molecules, which are built from different atoms. Corresponding examples reported in literature to date can be sub-categorized into two different geometries. First, those approaches based on structures exhibiting a $3 \mathrm{D}$ heterogeneous material composition, which gain their chiral optical response dominantly from their 3D chiral geometry rather than by their heterogeneity alone ${ }^{17,32,33}$. And second, a theoretical approach based on quasi-two-dimensional nanostructures exhibiting an inhomogeneous and asymmetric material composition, while also lacking symmetry with respect to their shape and geometry ${ }^{27}$. Both categories have been studied for arrays forming lattices or metasurfaces of the corresponding structures only.

In this Letter, we propose a truly planar nanostructure, which is geometrically achiral (mirror-symmetric) but exhibits a significant chiral optical response, that is, $\mathrm{CD}$, due to its heterogeneous composition. This response is observed for an individual nanostructure (as opposed to the arrangement in an array or a metasurface), which is embedded homogeneously into a dielectric medium and illuminated under normal incidence. This is in striking contrast to other two-dimensional chiral structures discussed in literature so far. In the present case, the structural symmetry is broken, and a handedness or chiral response is induced, by the heterogeneous material composition of the assembly. With respect to its geometry, the nanostructure, assembled from three equally sized achiral nanodisks, is super-imposable with its mirror-image by a simple rotation in two-dimensional space. In contrast, the trimer is not superimposable with its mirror image anymore if the heterogeneous material composition is taken into account. As we will show later on, it is, therefore, intrinsically chiral.

\section{Results}

Numerical results for trimers built from nanodisks. For our numerical study, we have chosen nanodisks made from gold $(\mathrm{Au})$ and silicon (Si). The spectra of individual Si nanodisks exhibit multiple resonances depending on the particle size, with the magnetic dipole representing the fundamental particle resonance. It becomes apparent that this behaviour is caused by the dielectric properties and the high refractive index of Si nanoparticles in the visible and near-infrared spectral range that has been studied extensively in the recent years ${ }^{34-38}$. The nanodisks have a diameter of $180 \mathrm{~nm}$ and a height of $30 \mathrm{~nm}$ embedded into a homogeneous environment with a refractive index of $n_{\text {air }}=1$ (see Fig. 1b,c). For excitation, we illuminate the trimer with a weakly focused Gaussian light beam (numerical aperture of $\mathrm{NA}=0.4$ ) propagating along the negative $z$-direction. This choice is also motivated by the fact that for this low NA, the structures are excited by a dominantly transverse electric field (plane-wave-like). The generation of longitudinal electric field components (which carry a phase vortex) upon tight focusing and their additional interaction with the trimer under study is, therefore, minimized ${ }^{39}$. It should be emphasized here again that we aim for studying individual trimeric structures only because we are interested in their intrinsic chiral optical response rather than collective effects caused by an arrangement of such structures in two-dimensional arrays or metasurfaces.

First, we present the spectra of individual trimers embedded in air for right- and left-handed circularly polarized (lhcp, rhcp) 

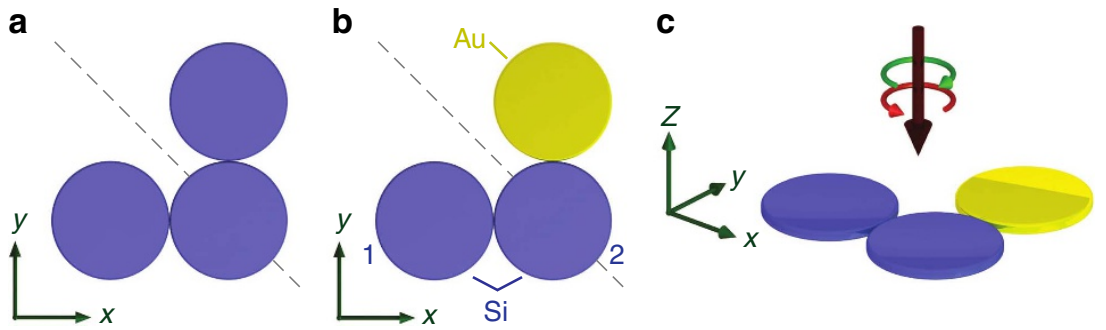

Figure 1 | Illustration of the investigated heterogeneously composed nanodisk trimer. (a) Sketch of a three-particle system (trimer) consisting of nanodisks of the same size and material all placed in the same plane. (b) If one of the particles at the end of the trimer is replaced by an equally sized disk made from a different material, the trimer still exhibits geometrical mirror-symmetry (grey dashed line indicates its geometric symmetry axis), but with respect to the material composition, its symmetry is broken. The trimeric nanostructure consists of three nanodisks, two made of silicon (blue) and one made of gold (yellow). (c) In the simulations, the trimer is illuminated with circularly polarized light (Gaussian beam; green arrow-right circular polarization, red arrow-left circular polarization), which impinges normally (along the negative $z$-direction) to the plane defined by the three disks (xy-plane). The thickness (height) and diameter of the disks were chosen to be 30 and $180 \mathrm{~nm}$, respectively.

excitation under normal incidence to underline the statement made above that individual trimers with a material-compositioninduced break of symmetry exhibit chiral properties (see transmittance in Fig. 2a and reflectance in Fig. 2b). The $\mathrm{CD}$ spectrum of the structure can be calculated as $\mathrm{CD}=$ $A_{\mathrm{lhcp}}-A_{\text {rhcp }}$ where $A=1-T-R$ is the absorbance, and $T$ and $R$ are the transmittance and reflectance, respectively. The $C D$ spectrum exhibits a maximum at a wavelength of $520 \mathrm{~nm}$ and is positive throughout the investigated spectrum, indicating a fixed optical handedness of the structure in this range (see Fig. 2c). By changing the order of the nanodisks in the trimer (exchanging the first Si disk (1) with the Au disk), the handedness of the trimer is inverted and the $\mathrm{CD}$ spectrum changes its sign (see also Supplementary Note 1 and Supplementary Fig. 1).

To understand the origin of this CD spectrum, we study the response of individual nanodisks. The resonance spectra of $\mathrm{Au}$ nanodisks for linearly polarized excitation (Fig. 2d,e; dotted cyan-coloured curve) are dominated by the electric dipole mode (vertical dotted line). On the other hand, the Si nanodisk exhibits no resonant features in the spectral range studied here (see also Fig. 2d,e; dotted-dashed red curve) when excited with linearly polarized light from the top. It can be shown that this is a simple consequence of the chosen height of the disk ${ }^{37}$. In contrast, the situation is considerably different for an excitation of the disk with light being linearly polarized and propagating in the $x y$-plane. This scenario mimics the case of light scattering in the plane of the nanodisks and exciting neighbouring particles in the trimer scheme. The corresponding spectra (see again Fig. 2d,e; dashed magenta-coloured curve) show a clear resonance at a wavelength of $520 \mathrm{~nm}$ (vertical dashed line). A detailed study of the electric and magnetic fields in the disk forming the resonant mode reveals that it can be associated with the fundamental magnetic dipole resonance formed by a curl of the electric field inside the $x y$-plane inside disk. This resonant mode cannot be excited directly by a linearly or circularly polarized light beam impinging along the $z$-axis, but it may be induced by the mutual interaction between neighbouring particles in the trimer. For the sake of completeness, we also include the resonance spectra of the heterogeneously composed trimer for $x$ - $\left(E_{x, \text { inc }}\right)$ and $y$-polarized $\left(E_{y, \text { inc }}\right)$ excitation in Fig. 2 d,e. The spectra for both excitations schemes differ drastically from each other, also indicating the material-induced asymmetry of the studied system.

In the trimeric arrangement, the transverse dipole moments of the individual disks can be excited (non-resonantly) both directly by the impinging circularly polarized light field and by light scattered off neighbouring particles. This leads to hybridization of the modes ${ }^{40,41}$. It is this hybridization of the disk modes for the excitation with $x$ - and $y$-polarized light that leads to different resonance spectra (see Fig. 2d,e), due to the chosen material-induced break of symmetry. In addition, the relative phase between the transverse electric field components $E_{x, \text { inc }}$ and $E_{y, \text { inc }}$ for circularly polarized excitation $( \pm \pi / 2)$ defines the phase between the induced transversely oriented dipole moments. The key to understanding the emerging optical chirality of the nanostructure at hand is the interaction between the modes excited directly by the incoming light field with the same modes excited via particle scattering or hybridization. Furthermore, also the interference between additional particle modes contributes, induced, for instance, in the Si disks solely by scattering of neighbouring particles for normal excitation. This phenomenon can be seen in Fig.2f, where the field distributions in a cut-plane through the trimeric disk assembly are plotted for both handedness of the excitation field and at a fixed wavelength of $520 \mathrm{~nm}$, for which the CD spectra peaked. The plotted fields exhibit different spatial distributions indicating that for both cases different particle modes contribute to the scattering process. Both, the plotted distributions of the instantaneous electric field (white arrows; transverse components shown only) and the modulus of the longitudinal magnetic field component $\left(\left|H_{z}\right|\right)$ indicate that for left-handed circularly polarized excitation (Fig. 2f, left), a longitudinal magnetic dipole component is observable dominating the mode in the central Si particle (2). Consequently, the coupling of the particles with each other induces an additional dipole contribution. In the other Si disk (1), the mode is more complex. For right-handed circularly polarized light (Fig. 2f, right), the situation is different. The mode structure in the central Si disk is also more complex and does not exhibit a clear fingerprint of a longitudinal magnetic dipole moment anymore.

Not less important for this discussion is the fact that even though also the same particle modes (transverse dipoles) might be involved in the optical response of the trimer for both handednesses of excitation, they will differ in their multipole amplitudes due to the presence of the Au disk and, hence, contribute with different strengths. Ultimately, those modes excited by scattering can be different for the $x$ - and $y$-field components of the impinging circularly polarized light beam (see Fig. 3), due to the symmetry and geometry of the trimer. This causes an additional phase delay between the directly excited and scattering-induced particle modes. They can, therefore, interfere with each other coherently, resulting in a suppression or enhancement of such particle modes, which ultimately depends on the relative phase between the components of the excitation field or, equivalently, on the handedness of the excitation. 


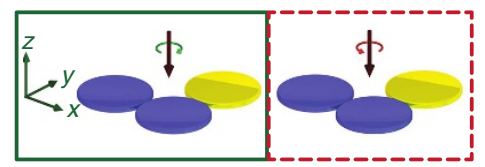

a

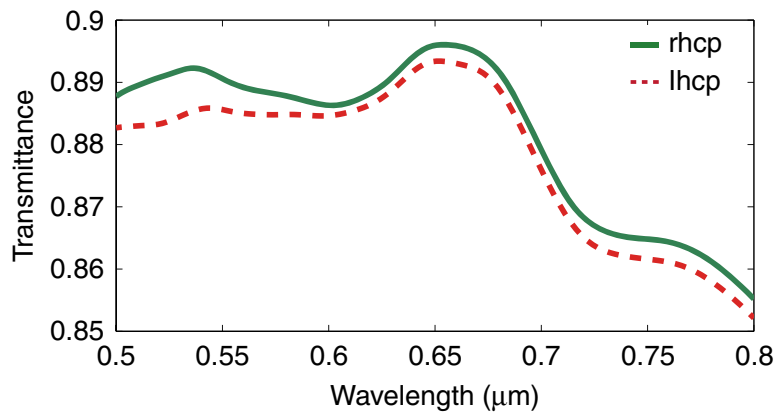

b

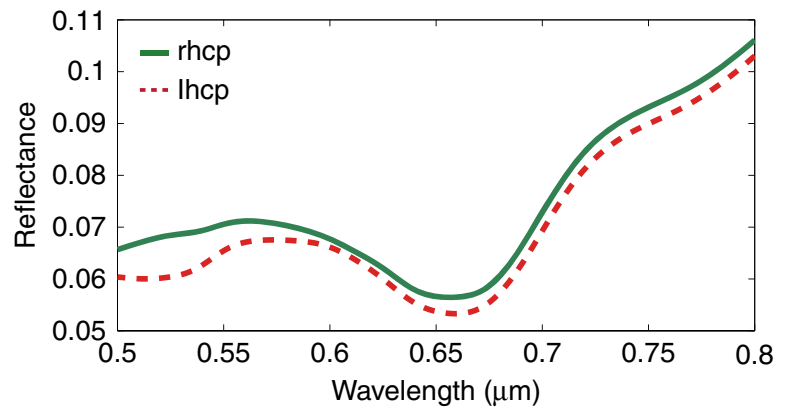

C

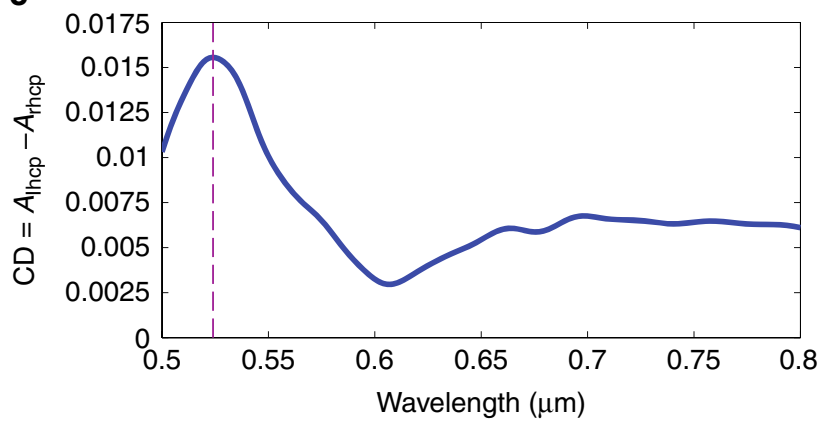

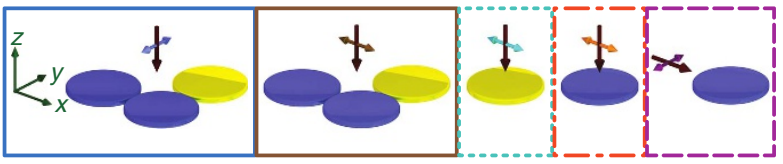

d

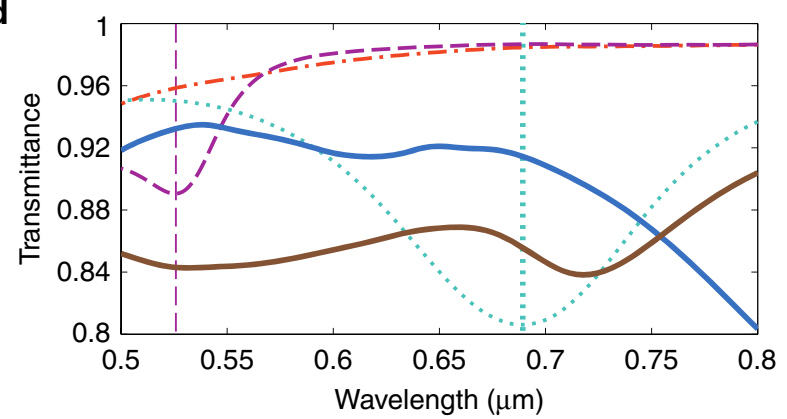

e

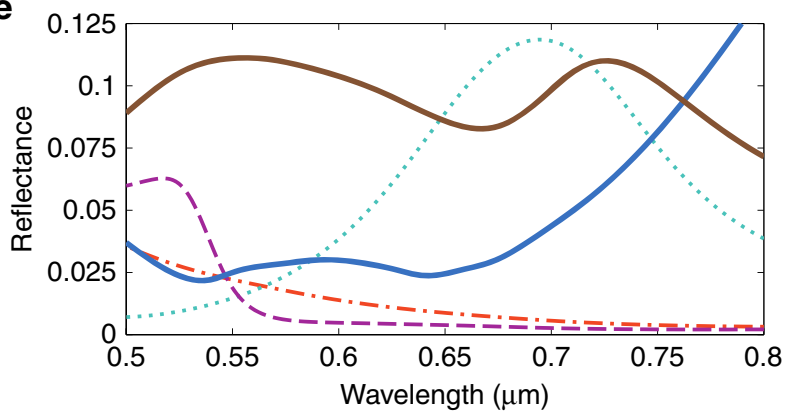

$\mathbf{f}$
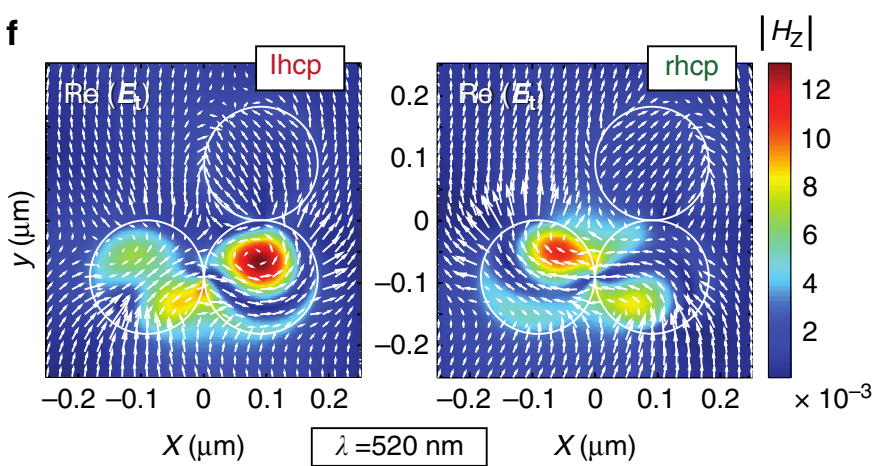

Figure 2 | Numerical results for different nanodisks system. Numerically calculated (a) transmittance and (b) reflectance spectra for an individual nanodisk trimer for left-handed circularly polarized (Ihcp, dashed red line) and right-handed circularly polarized (rhcp; solid green line) light excitation. (c) Resulting circular dichroism (CD) curve. (d) Transmittance and (e) reflectance spectra for a trimer (solid blue and brown curves) and individual nanodisks (dotted and dashed curves) for linearly polarized excitation. The corresponding excitation schemes are indicated above (d). The vertical dotted cyan-coloured line indicates the spectral position of the electric dipole resonance of an individual Au nanodisk, whereas the vertical dashed magenta-coloured line marks the position of the magnetic dipole resonance of an individual Si nanodisk for the indicated excitation schemes.

(f) Distributions of the instantaneous in-plane electric field $\left(\mathbf{E}_{t}=\left(E_{x}, E_{y}\right)\right.$; white arrows) and the modulus of the longitudinal magnetic field component $\left(\left|H_{z}\right|\right.$; colour-coded) for a wavelength of $520 \mathrm{~nm}$ plotted in a plane parallel to the $x y$-plane cutting through the centers of the disks.

The aforementioned arguments become more apparent when looking at the induced field patterns for $x$ - and $y$-polarized excitation as shown exemplarily in Fig. 3 again for a wavelength of $520 \mathrm{~nm}$. This field-based analysis also enables a more quantitative discussion of the material-composition-induced chirality proposed here. In Fig. 3a, the time evolution of the instantaneous longitudinal magnetic field component $\left(H_{z}\right)$ is plotted in steps of $\pi / 2$ for an excitation with a x-polarized Gaussian beam. For comparison, the corresponding numerical results for the same trimer excited with $y$-polarized light are shown in Fig. 3b. For $x$-polarized excitation, the field distributions inside the Si nanodisks resemble a two-lobe pattern, which is a characteristic fingerprint of the field created by an electric dipole mode oscillating along the $x$ axis. The actual electric dipole resonances of the individual Si nanodisks are found outside the investigated spectral range. Hence, the excited mode is driven off-resonance. The distributions are altered by the nearfield interaction of both particles, indicating mode hybridization. As time evolves, the magnetic field oscillates, preserving the initial distributions of the field structure throughout a full period, which are similar and in-phase for both Si disks. For symmetry reasons, the scattering and near-fields of the Si particle at the lower left position (1) of the trimer cannot efficiently excite a resonant longitudinal magnetic dipole moment (along the $z$ axis) in the second $\mathrm{Si}$ particle or vice versa, because its field is dominantly polarized along the $\mathrm{Si}$-Si-dimer axis. In this context, the contribution of the scattering off the Au particle to the field structure is minor, because it is driven far off-resonance. 
a

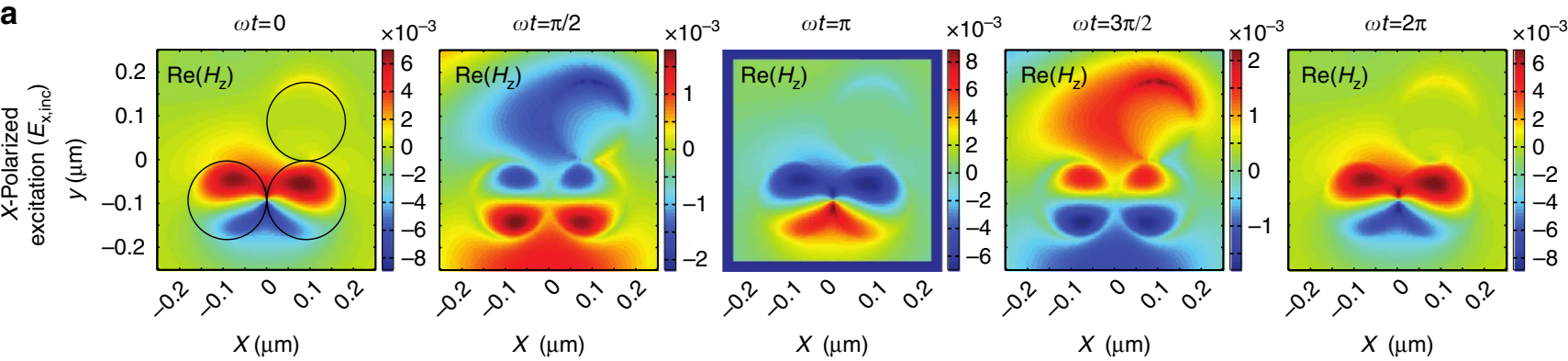

b
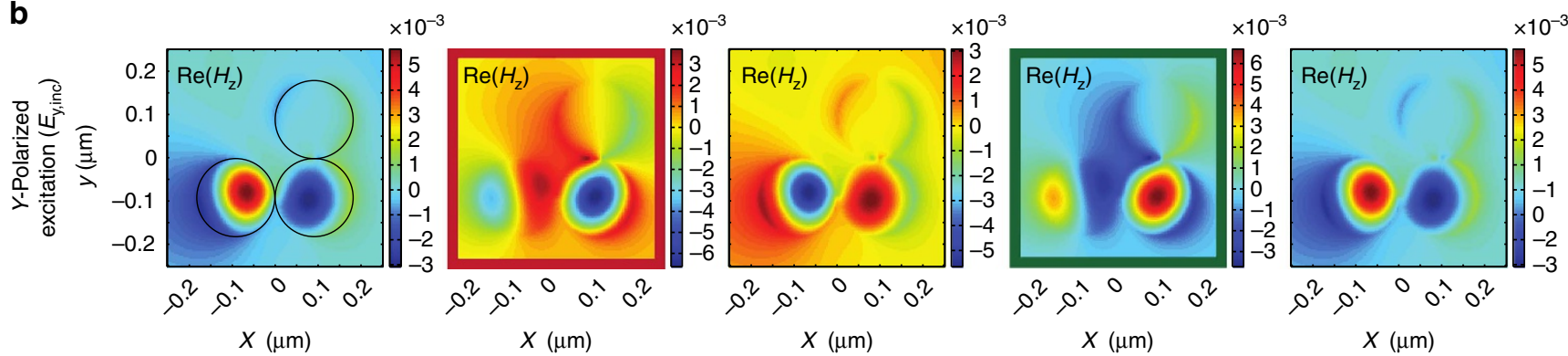

Figure 3 | Near-field maps for different excitation schemes. Time-evolution of the longitudinal magnetic field component $\operatorname{Re}\left(H_{z}\right)$ plotted in a plane parallel to the $x y$-plane cutting through the centers of all particles for two different excitation schemes and a fixed wavelength of $520 \mathrm{~nm}$. (a) Excitation with an $x$-polarized Gaussian light beam. (b) Excitation with a y-polarized Gaussian light beam. Each field map is normalized to its individual minima and maxima resulting in different colour-scales for different maps. The geometrical outlines of the three nanosdisks (top: Au; bottom: Si) are indicated by black circles. For right-handed (left-handed) circularly polarized excitation, the frames indicated in blue in $\mathbf{a}$ and green (red) in $\mathbf{b}$ overlap temporally.

In contrast to the excitation with $x$-polarization $\left(\mathbf{E}_{\text {inc }}=\left(E_{x, \text { inc }}, 0,0\right)\right)$, the field patterns in both Si disks differ strongly when excited with $y$-polarized light $\left(\mathbf{E}_{\text {inc }}=\left(0, E_{y, \text { inc }}, 0\right)\right)$ (see Fig. 3b). For this case, the field distribution inside the central Si particle (2) exhibits only one strong and dominant lobe, which oscillates in time parallel to the $z$ axis. The corresponding electric field shows a curl-like distribution. This field pattern corresponds to that of a longitudinally oscillating magnetic dipole moment, which, for an individual disk, is resonantly excited at the chosen wavelength (see Fig. 2d,e). It is induced by the vertically $(y)$ oscillating transverse electric dipole mode in the neighbouring $\mathrm{Si}$ disk (1), excited directly by the incoming light field (see, for instance, second image in Fig. 3b). The same holds true for the vertically oriented electric dipole moment excited in the central Si particle (2), inducing a magnetic dipole moment in the left-hand Si disk (1). Nonetheless, the time evolution of the fields within both disks is not the same, which is a consequence of the presence of the Au particle and, thus, a result of the material-induced break of symmetry. It should be mentioned here, that beside the coupling of the longitudinal magnetic to the transverse electric dipole moments of the Si disks, also the electric dipole moments of both Si nanodisks themselves are coupled to each other ${ }^{40}$.

After this analysis it becomes clear, why the field distributions for right- and left-handed circularly polarized excitation as shown in Fig. $2 \mathrm{f}$ differ from each other drastically and why they are not symmetric, as they would be for a simple $\mathrm{Si}$ dimer. For right-handed and left-handed circularly polarized light, the incoming beam can be written as $\mathbf{E}_{\text {inc }}=\left(E_{x, \text { inc }},-i E_{y, \text { inc }}, 0\right)$ and $\mathbf{E}_{\text {inc }}=\left(E_{x, \text { inc }}, i E_{y, \text { inc }}, 0\right)$, respectively. As a consequence of the excitation with circularly polarized light, the two temporal field evolutions shown in Fig. 3a,b have to be shifted relative to each other due to an additional relative phase-delay between the corresponding field distributions of $\mp \pi / 2$. As a result, the frames indicated in blue in Fig. $3 a$ and green in Fig. $3 b$ overlap temporally for right-handed circularly polarized excitation, whereas the blue frame overlaps with the red frame for left-hand circular polarization. The corresponding field distributions in Fig. 3a,b, therefore, interfere with each other forming the patterns shown in Fig. 2f. The observed asymmetries between the near-fields of the individual heterogeneous trimer are consequently a result of constructive and destructive interference of the symmetric and asymmetric field patterns in Fig. 3a,b. Ultimately, this interference results in different resonance spectra for excitation with different handedness of the incoming light.

Experimental results for trimers built from nanospheres. For the experimental demonstration of the proposed scheme, we choose a nanotrimer assembled from individual nanospheres (see Fig. 4a) rather than nanodisks as studied numerically above. As can be seen from Fig. $4 \mathrm{a}$, the particle diameters vary slighly for the $\mathrm{Au}(171 \mathrm{~nm})$ and $\mathrm{Si}$ (both $188 \mathrm{~nm}$ ) particles. Also a tiny gap between the $\mathrm{Au}$ and $\mathrm{Si}$ particles can be observed. We will see later on that this only marginally influences the results. We use a pick-and-place-based approach for the assembly of spherical nanoparticles, realized by operating an atomic force microscope (AFM) inside the vacuum-chamber of a scanning electron microscope $(\mathrm{SEM})^{42,43}$ (see also Methods section for more details). Because we are interested in the optical measurement of a single trimer only, the pick-and-place-based fabrication is straight-forward and time-efficient. A fabrication of trimers consisting of nanodisks rather than nanospheres would require multiple complex and time-consuming coating and lithography steps, which is very inconvenient for the investigation of the proposed scheme and the proof of its principle. The scheme involving nanodisks as discussed above enabled a more fundamental discussion and intuitive understanding of the effects causing a chiral behaviour of heterogeneous geometrically symmetric trimers. Nonetheless, the underlying physics are similar for both systems (disks and spheres).

To be able to study an individual nanotrimer experimentally, we utilize a custom-built optical set-up ${ }^{44}$. A sketch of the system 


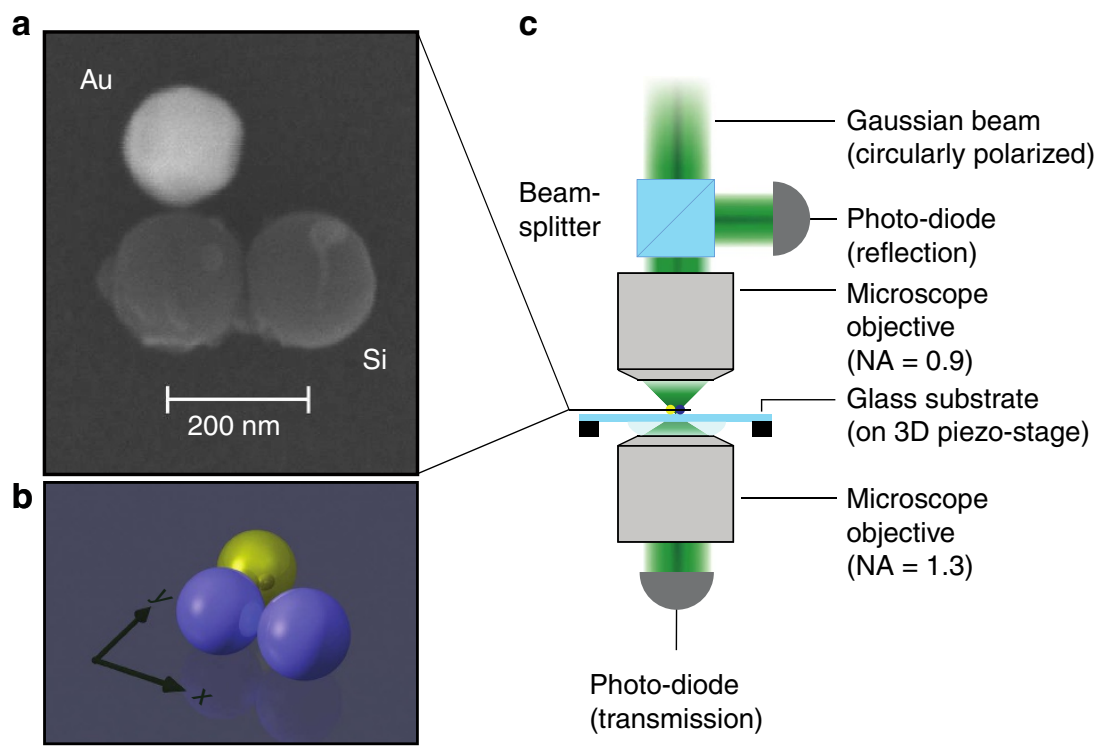

Figure 4 | Investigated nanostructure and experimental set-up. (a) SEM micrograph of the investigated trimer assembled from one Au (white; diameter $\approx 171 \mathrm{~nm}$ ) and two Si (dark grey; diameters $\approx 188 \mathrm{~nm}$ ) nanospheres on a borosilicate glass (BK7) substrate. (b) Sketch of the trimer and chosen coordinate frame. It should be noted here that the investigated trimer exhibits a mirrored geometry with respect to the nanodisk trimer discussed before. (c) Simplified sketch of the experimental set-up utilized for the measurement of individual nanosphere trimers. A circularly polarized fundamental Gaussian light beam enters a microscope objective (MO) with a high numerical (NA) of 0.9. The entrance aperture of the $\mathrm{MO}$ is not filled, hence reducing the effective NA to $\sim 0.4$. The focused light beam has a diameter of $\sim 0.8 \mu \mathrm{m}$ (full-width at half-maximum) at a wavelength of $625 \mathrm{~nm}$. The sample composed of a substrate with individual nanotrimers assembled on top is mounted on a 3D-piezo-stage, enabling precise positioning of the structure relative to the beam. A second immersion-type $\mathrm{MO}$ with an NA of 1.3 is located below the sample to collect the light in the forward direction, which is detected with a photo-diode. Light reflected or scattered backwards is collected by the upper $\mathrm{MO}$ and guided to a second photo-diode by a set of non-polarizing beamsplitters.

is shown in Fig. 4c (see Methods section for details). For the measurements, the wavelength is selected with the spectral filter, the nanotrimer is placed in the focal plane and it is scanned line-by-line through the focal spot several times. From such measurements, the transmitted and reflected power values for the particle sitting effectively on the optical axis in the focal plane are retrieved and averaged. The corresponding data is normalized to the power transmitted through and reflected from the air-substrate interface, respectively. Following this procedure, the measurements are repeated for different wavelengths and for both handednesses of the circularly polarized input beam, resulting in the corresponding normalized resonance spectra in the spectral range from 480 to $750 \mathrm{~nm}$ as shown in Fig. 5a,b. The figure also includes the simulation results for the investigated scheme (see Fig. 5d,e), taking into account the experimental conditions, such as the BK7 substrate and the beam configuration. For the sake of convenience, we set the diameter of all particles to $180 \mathrm{~nm}$ and the inter-particle gap to $0 \mathrm{~nm}$ in the simulations. Furthermore, we also incorporate a thin $\mathrm{SiO}_{2}$ surface layer around the $\mathrm{Si}$ nanospheres naturally occurring due to oxidation. The layer thickness is estimated to be approximately $8 \mathrm{~nm}$ (ref. 38). The actual diameter of the Si core is therefore reduced correspondingly. In the experiment, a thin layer of aluminum-doped zinc oxide (AZO; $6 \mathrm{~nm}$ ) between the substrate and the trimer was used to suppress charging effects during the pick-and-place fabrication. This layer is not taken into account in the simulations. The experimental data is normalized to the transmission through and reflection at the AZO-on-glass substrate, whereas the numerical data was retrieved and normalized for a glass substrate only.

From both sets of data, we calculate the spectral distributions of the resulting $C D$ spectrum, displayed in Fig. 5c,f for the experimental and numerical results, respectively. The experimental reflectance and transmittance curves in Fig. 5a,b bear striking similarities with the spectra retrieved from numerical calculations (see Fig. 5d,e). The slight red-shift of the experimental data with respect to the numerical results and also the different absolute values for transmittance and reflectance data sets are caused by the presence of the aforementioned AZO-layer on the experimental sample. Nonetheless, both data sets underline the chiral behaviour of the geometrically mirror-symmetric particle ensemble (trimer). Apparently, these spectra are more rich in comparison to those of trimers composed of nanodisks discussed above. This is caused by the fact that for nanospheres of the chosen size made from high refractive index dielectrics such as $\mathrm{Si}$ in the visible or near-infrared spectral range, magnetic and electric dipole resonances appear at wavelengths in the investigated spectral range. Therefore, the incoming light field can now also resonantly excite a magnetic dipole-a transversely oscillating magnetic dipole moment perpendicular to the $z$ axis-in the investigated spectral range, which contributes to the resonance behaviour of the trimer. Furthermore, the utilized substrate as a support for the particles or, in other words, the air-dielectric interface also influences the retrieved spectra. As discussed before, the substrate additionally breaks the forward-backward symmetry of the system, further influencing its chiral response. Nonetheless, the trimer assembled from nanospheres as investigated here of course also shows a chiral response if embedded in a homogeneous environment, as it was also the case for the disks studied above. It is also worth noting that the corresponding CD spectra for the investigated trimer made from nanospheres show a change of sign in certain spectral regions. Hence, the actual optical handedness of the trimer also changes and, therefore, depends on the wavelength. For comparison, we also assembled and measured a nanotrimer with opposite handedness resulting in an inversion of the CD spectra (see Supplementary Note 1 and Supplementary Fig. 1). Also, we show (see Supplementary Note 1 and Supplementary Fig. 2) the 


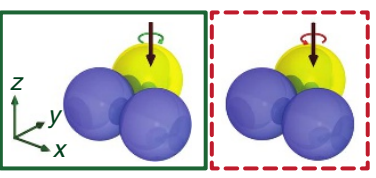

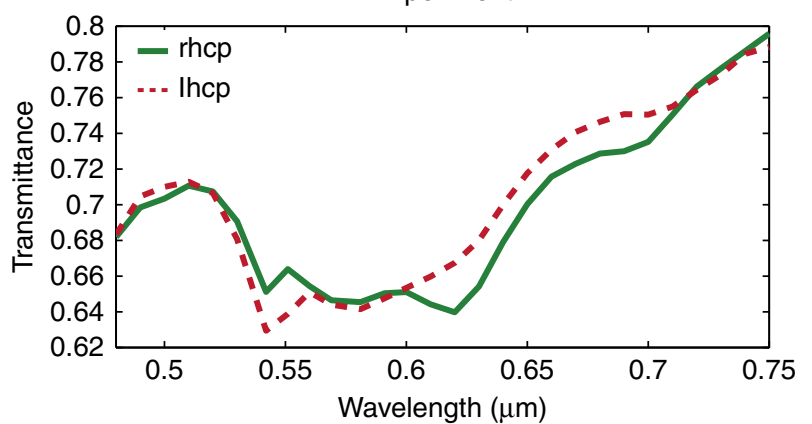

b
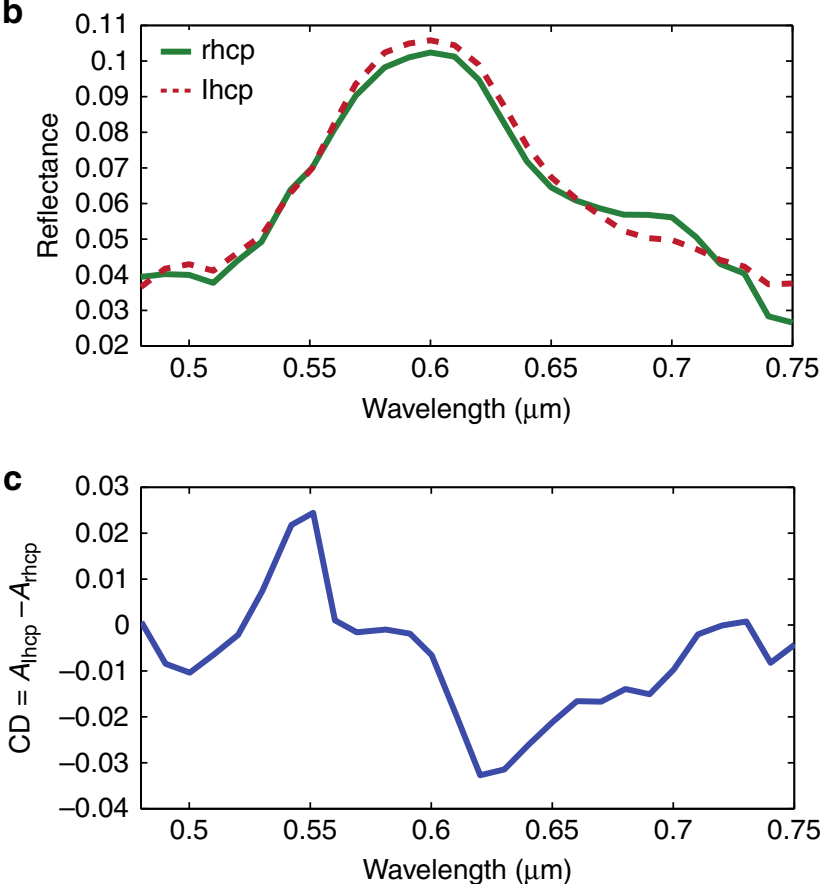

d
Simlulation

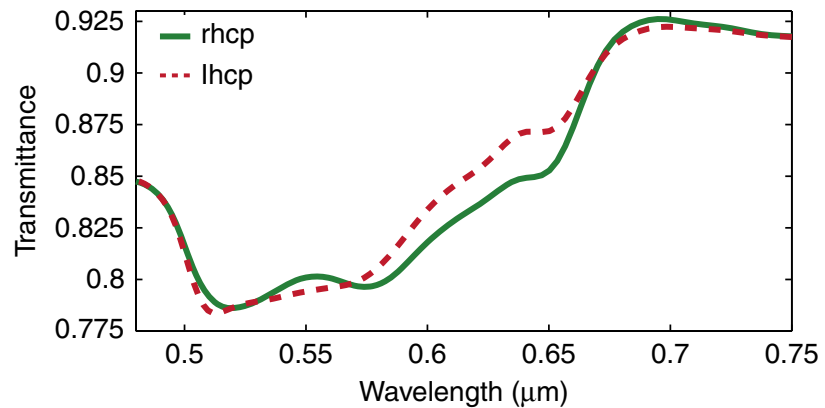

e

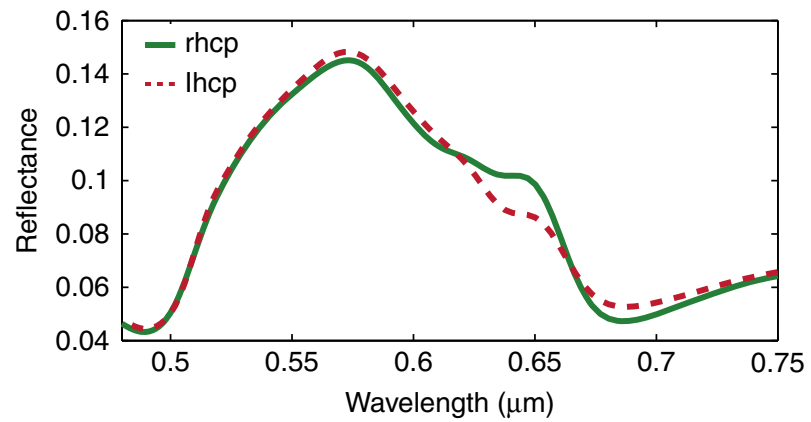

$\mathbf{f}$

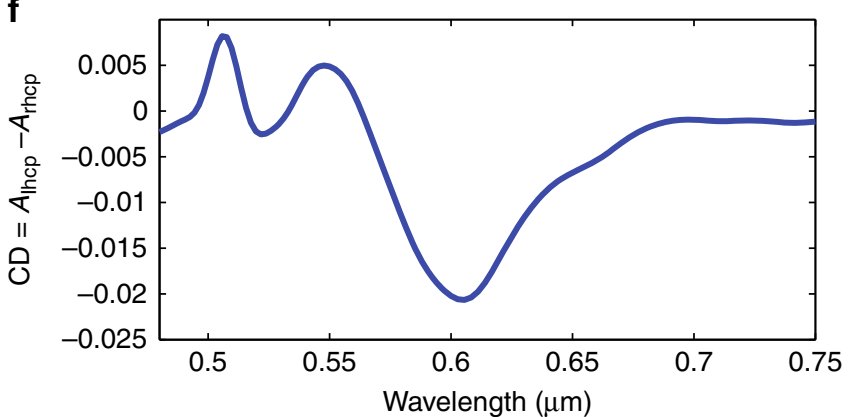

Figure 5 | Comparison of experimental and numerical results for a heterogeneous nanosphere trimer. (a-c) Experimental and (d-f) numerical results for an individual trimer assembled from Au and Si nanospheres on a glass substrate (see Fig. 4). Transmittance and reflectance as well as the CD spectra are shown. In the experiment, a thin layer of AZO $(6 \mathrm{~nm})$ was present on top of the glass substrate to prevent charging effects during fabrication.

experimental and numerical results of a measurement on a second trimer exhibiting the same handedness like the trimer discussed above.

The observed CD spectra can be further modified easily by assembling trimers from smaller or larger nanopheres, by changing the size of the central particle relative to the other particles, still keeping the trimer geometrically symmetric, or by using nanospheres made from materials different than $\mathrm{Au}$ and Si. These approaches allow for tuning the particle spectra relative to each other. For instance for larger particles, also higher order resonances contribute to the scattering and the inter-particle coupling, enhancing the performance of the chiral particle ensemble. It is also conceivable that the trimer may be composed of equally sized particles of three different materials or from more than just three particles. These design criteria allow for a broad range of structural arrangements, further tuning and boosting the material-composition-induced chiral effect discussed above.

\section{Discussion}

In conclusion, we have introduced and studied-as a proof-ofprinciple-an approach for the realization of optically chiral nanostructures. The proposed scheme is based on a geometrically achiral nanoparticle (sphere, disks or similar) assembly, which nonetheless exhibits a chiral optical response due to its heterogeneous composition. The chiral behaviour of the trimer is caused by the mutual interaction of neighbouring particles when excited under normal incidence. To demonstrate this phenomenon also experimentally, we have fabricated heterogeneous trimers from individual $\mathrm{Au}$ and $\mathrm{Si}$ nanospheres on a substrate, using a highly precise pick-and-place technique realized by operating an AFM inside the vacuum chamber of an SEM. Such heterogeneously-composed particle ensembles allow for a high tunability with respect to their spectra and chiral optical response. With the possibility of tailoring the multipolar response of the individual constituents, the response of the chiral assemblies can be tuned in a straight-forward fashion over a 
broad spectral range. Furthermore, we believe that based on this material composition-enabled approach to chirality of nanostructures introduced and discussed in this Letter, also large-scale chiral materials and metasurfaces can be designed to allow for the realization of ultra-thin chiral filters and polarizers. Potential approaches for fabricating large arrays of individual heterogeneous nanoparticle assemblies could be based on the methods recently described in literature ${ }^{17,45}$. It is worth noting that based on the arguments discussed above, some of the trimeric particle arrangements presented in ref. 45 should also show a chiral optical behaviour.

\section{Methods}

Sample fabrication. The metal particles that were used for this study are citrate-stabilized colloidal Au nanoparticles from BBI Solutions dispersed in water. A drop of such a colloidal solution was deposited on a clean silicon substrate and left for $2 \mathrm{~h}$ to sediment. Afterwards, the remaining solution was decanted and the sample left to dry to serve as a reservoir sample for Au nanoparticles. The Si nanoparticles were obtained as a dry Si nanopowder from Materials Tech. Intl. The silicon nanopowder was dispersed in ethanol by ultrasonic treatment. A drop of this solution was then deposited onto a clean silicon sample as well and left to fully dry out to provide a reservoir sample with Si nanoparticles.

For the actual fabrication of individual heterogeneously composed trimers we then applied a pick-and-place-based manipulation approach. This method is based on operating an AFM inside the vacuum-chamber of an $\mathrm{SEM}^{42,43}$. In this custombuilt system, the AFM tip is modified to permit precise handling of nanoparticles. This approach allows for choosing particles with the desired size, shape and material from the aforementioned particle reservoirs and enables precise positioning of the individual particles relative to each other on a substrate, taking also advantage of the attractive forces between particles at the nanoscale. To prevent charging of the substrate resulting from the imaging process of the sample with the electron beam while manipulating the nanoparticles, a thin AZO-layer $(6 \mathrm{~nm})$ is deposited on the borosilicate glass (BK7) substrate first.

Experimental set-up and measurement scheme. The custom-built optical set-up (see Fig. 4c) consists of a spectral filter (acousto-optical spectral filter; spectral bandwidth: $1 \mathrm{~nm}$ ) placed behind the output of a broadband fiber-based light source. It selects the central wavelength of the transmitted Gaussian light beam. The light beam is transmitted through a quarter-wave plate. The resulting circularly polarized beam is guided top-down into a microscope objective with a high NA and focused onto the sample. The size of the input beam is chosen such that the input beam does not fill the entrance aperture of the microscope objective. This way, an effectively smaller focusing NA (0.4) is achieved and the influence of longitudinal electric field components created upon strong focusing is reduced ${ }^{39}$. The sample is composed of a borosilicate glass (BK7) substrate with a thickness of $\sim 170 \mu \mathrm{m}$ and a thin AZO-layer $(6 \mathrm{~nm})$ on top. On the surface of the substrate, individual trimeric structures are assembled using the above-mentioned pick-and-place handling technique. Figure 4a shows an SEM micrograph of the assembly investigated here. The sample is mounted on a piezo-stage, which allows for precisely positioning it relative to the excitation beam. Light transmitted through the substrate or scattered in the forward direction by the trimer is collected by a second microscope objective. This oil-immersion objective with an NA of 1.3 collimates the collected light. The resulting beam is guided to a photodetector. Light reflected from the air-substrate interface or back-scattered light is collected within a large solid angle by the upper objective, originally used for focusing the incoming light beam. A non-polarizing beamsplitter inserted in front of this focusing objective allows for separating the incoming and a part of the reflected light beam and guides the latter to a detector. For guaranteeing a high quality of the input and the reflected light beams with respect to their polarization, we utilize a set of beam-splitters, all non-polarizing in type, for preserving the polarization state ${ }^{46,47}$

Data availability. The data that support the findings of this study are available from the corresponding authors upon request.

\section{References}

1. Gansel, J. K. et al. Gold helix photonic metamaterial as broadband circular polarizer. Science 325, 1513-1515 (2009).

2. Thiel, M., Fischer, H., von Freymann, G. \& Wegener, M. Three-dimensional chiral photonic superlattices. Opt. Lett. 35, 166-168 (2010).

3. Höflich, K., Yang, R. B., Berger, A., Leuchs, G. \& Christiansen, S. The direct writing of plasmonic gold nanostructures by electron-beam-induced deposition. Adv. Mater. 23, 2657-2661 (2011).

4. Helgert, C. et al. Chiral metamaterial composed of three-dimensional plasmonic nanostructures. Nano Lett. 11, 4400-4404 (2011).
5. Frank, B. et al. Large-area 3D chiral plasmonic structures. ACS Nano 7, 6321-6329 (2013).

6. Belardini, A. et al. Second harmonic generation circular dichroism from self-ordered hybrid plasmonic-photonic nanosurfaces. Adv. Opt. Mater. 2 208-213 (2014).

7. Esposito, M. et al. Nanoscale $3 \mathrm{~d}$ chiral plasmonic helices with circular dichroism at visible frequencies. ACS Photon. 2, 105-114 (2015).

8. Schäferling, M., Dregely, D., Hentschel, M. \& Giessen, H. Tailoring enhanced optical chirality: design principles for chiral plasmonic nanostructures. Phys. Rev. X 2, 031010 (2012).

9. Valev, V. K., Baumberg, J. J., Sibilia, C. \& Verbiest, T. Chirality and chiroptical effects in plasmonic nanostructures: fundamentals, recent progress, and outlook. Adv. Mater. 25, 2517-2534 (2013).

10. Liu, H. et al. Magnetic plasmon hybridization and optical activity at optical frequencies in metallic nanostructures. Phys. Rev. B 76, 073101 (2007).

11. Liu, N., Liu, H., Zhu, S. \& Giessen, H. Stereometamaterials. Nat. Photon. 3, 157-162 (2009)

12. Decker, M. et al. Strong optical activity from twisted-cross photonic metamaterials. Opt. Lett. 34, 2501-2503 (2009).

13. Fan, Z. \& Govorov, A. O. Plasmonic circular dichroism of chiral metal nanoparticle assemblies. Nano Lett. 10, 2580-2587 (2010).

14. Kuzyk, A. et al. DNA-based self-assembly of chiral plasmonic nanostructures with tailored optical response. Nature 483, 311-314 (2012).

15. Hentschel, M., Schäferling, M., Metzger, B. \& Giessen, H. Plasmonic diastereomers: adding up chiral centers. Nano Lett. 13, 600-606 (2013).

16. Shen, X. et al. Three-dimensional plasmonic chiral tetramers assembled by DNA origami. Nano Lett. 13, 2128-2133 (2013).

17. Ogier, R., Fang, Y., Svedendahl, M., Johansson, P. \& Käll, M. Macroscopic layers of chiral plasmonic nanoparticle oligomers from colloidal lithography. ACS Photon. 1, 1074-1081 (2014).

18. Papakostas, A. et al. Optical manifestations of planar chirality. Phys. Rev. Lett. 90, 107404 (2003).

19. Rogacheva, A. V., Fedotov, V. A., Schwanecke, A. S. \& Zheludev, N. I. Giant gyrotropy due to electromagnetic-field coupling in a bilayered chiral structure Phys. Rev. Lett. 97, 177401 (2006).

20. Reichelt, M. et al. Broken enantiomeric symmetry for electromagnetic waves interacting with planar chiral nanostructures. Appl. Phys. B 84, 97-101 (2006)

21. Decker, M., Klein, M. W., Wegener, M. \& Linden, S. Circular dichroism of planar chiral magnetic metamaterials. Opt. Lett. 32, 856-858 (2007).

22. Plum, E., Fedotov, V. A. \& Zheludev, N. I. Extrinsic electromagnetic chirality in metamaterials. J. Opt. A: Pure Appl. Opt. 11, 074009 (2009).

23. Hendry, E. et al. Ultrasensitive detection and characterization of biomolecules using superchiral fields. Nat. Nanotechnol. 5, 783-787 (2010).

24. Zhao, R., Zhang, L., Zhou, J., Koschny, T. \& Soukoulis, C. M. Conjugated gammadion chiral metamaterial with uniaxial optical activity and negative refractive index. Phys. Rev. B 83, 035105 (2011).

25. Eftekhari, F. \& Davis, T. J. Strong chiral optical response from planar arrays of subwavelength metallic structures supporting surface plasmon resonances. Phys. Rev. B 86, 075428 (2012)

26. Narushima, T., Hashiyada, S. \& Okamoto, H. Nanoscopic study on developing optical activity with increasing chirality for two-dimensional metal nanostructures. ACS Photon. 1, 732-738 (2014).

27. Alali, F., Kim, Y. H., Baev, A. \& Furlani, E. P. Plasmon-enhanced metasurfaces for controlling optical polarization. ACS Photon. 1, 507-515 (2014).

28. Plum, E., Fedotov, V. A. \& Zheludev, N. I. Optical activity in extrinsically chiral metamaterial. Appl. Phys. Lett. 93 191911-1-3 (2008).

29. Plum, E. et al. Metamaterials: optical activity without chirality. Phys. Rev. Lett 102, 113902 (2009).

30. Sersic, I., Tuambilangana, C., Kampfrath, T. \& Koenderink, A. F. Magnetoelectric point scattering theory for metamaterial scatterers. Phys. Rev. B 83, 245102 (2011).

31. De Leon, I. et al. Strong, spectrally-tunable chirality in diffractive metasurfaces. Sci. Rep. 5, 13034 (2015).

32. Yeom, B. et al. Chiral plasmonic nanostructures on achiral nanopillars. Nano Lett. 13, 5277-5283 (2013)

33. Hentschel, M., Weiss, T., Bagheri, S. \& Giessen, H. Babinet to the half: coupling of solid and inverse plasmonic structures. Nano Lett. 13, 4428-4433 (2013).

34. Evlyukhin, A. B., Reinhardt, C., Seidel, A., Luk'yanchuk, B. S. \& Chichkov, B. N. Optical response features of Si-nanoparticle arrays. Phys. Rev. B 82, 045404 (2010).

35. García-Etxarri, A. et al. Strong magnetic response of submicron silicon particles in the infrared. Opt. Express 19, 4815-4826 (2011).

36. Kuznetsov, A. I., Miroshnichenko, A. E., Fu, Y. H., Zhang, J. \& Luk'yanchuk, B. Magnetic light. Sci. Rep. 2 (2012).

37. van de Groep, J. \& Polman, A. Designing dielectric resonators on substrates: combining magnetic and electric resonances. Opt. Express 21, 26285-26302 (2013).

38. Woźniak, P., Banzer, P. \& Leuchs, G. Selective switching of individual multipole resonances in single dielectric nanoparticles. Laser Photon. Rev. 9, 231-240 (2015). 
39. Zhao, Y., Edgar, J. S., Jeffries, G. D. M., McGloin, D. \& Chiu, D. T. Spin-to-orbital angular momentum conversion in a strongly focused optical beam. Phys. Rev. Lett. 99, 073901 (2007).

40. Albella, P. et al. Low-loss electric and magnetic field-enhanced spectroscopy with subwave-length silicon dimers. J. Phys. Chem. C 117, 13573-13584 (2013).

41. Bakker, R. M. et al. Magnetic and electric hotspots with silicon nanodimers. Nano Lett. 15, 2137-2142 (2015).

42. Bartenwerfer, M. et al.in 2011 IEEE International Conference on Mechatronics and Automation 171-176 (Beijing, China, 2011).

43. Mick, U., Banzer, P., Christiansen, S. \& Leuchs, G. in CLEO: 2014, STu1H.1 (Optical Society of America, 2014).

44. Banzer, P., Peschel, U., Quabis, S. \& Leuchs, G. On the experimental investigation of the electric and magnetic response of a single nano-structure. Opt. Express 18, 10905-10923 (2010).

45. Yang, A. et al. Hetero-oligomer nanoparticle arrays for plasmon-enhanced hydrogen sensing. ACS Nano 8, 7639-7647 (2014).

46. Tidwell, S. C., Ford, D. H. \& Kimura, W. D. Transporting and focusing radially polarized laser beams. Opt. Eng. 31, 1527-1531 (1992).

47. Zhan, Q. Vectorial Optical Fields-Fundamentals and Applications (World Scientific, 2014).

\section{Acknowledgements}

P.B. acknowledges financial support provided by the Alexander von Humboldt Foundation and the Canada Excellence Research Chair (CERC) in Quantum Nonlinear Optics. U.M. acknowledges financial support by the DFG via the Research Training Group GRK1896. The authors acknowledge fruitful discussions with S. Orlov.

\section{Author contributions}

P.B. conceived the idea; P.B. designed the structure; P.B. and P.W. performed the numerical simulations; U.M. fabricated the sample; P.W. performed the experiment; P.B. and I.D.L. analysed the data; R.W.B. and P.B. supervised all aspects of the project. All authors contributed to the preparation of the manuscript.

\section{Additional information}

Supplementary Information accompanies this paper at http://www.nature.com/ naturecommunications

Competing financial interests: The authors declare no competing financial interests.

Reprints and permission information is available online at http://npg.nature.com/ reprintsandpermissions/

How to cite this article: Banzer, P. et al. Chiral optical response of planar and symmetric nanotrimers enabled by heteromaterial selection. Nat. Commun. 7, 13117 doi: 10.1038/ncomms13117 (2016).

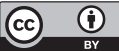

This work is licensed under a Creative Commons Attribution 4.0 International License. The images or other third party material in this article are included in the article's Creative Commons license, unless indicated otherwise in the credit line; if the material is not included under the Creative Commons license, users will need to obtain permission from the license holder to reproduce the material. To view a copy of this license, visit http://creativecommons.org/licenses/by/4.0/

(C) The Author(s) 2016 